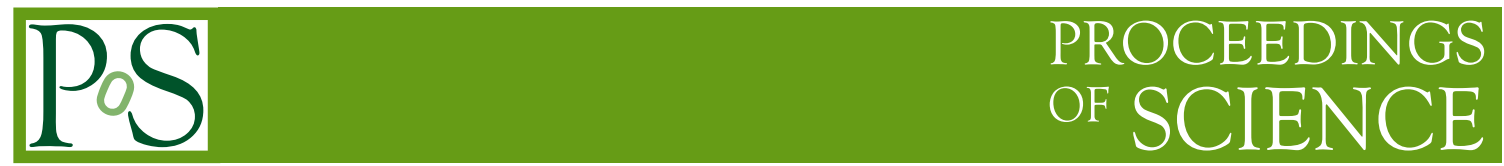

\title{
Measurements of diffraction in p-p collisions in CMS
}

\section{Robert CIESIELSKI*}

On behalf of the CMS Collaboration

( Rockefeller University (US))

E-mail: robert.ciesielski@rockefeller.edu, robertc@cern.ch

\begin{abstract}
We present measurements of soft and hard diffraction at the LHC, including new measurements of inclusive single- and double- diffractive cross sections, as well as forward rapidity gap cross section, and measurements of diffractive dijets at $7 \mathrm{TeV}$. We also show the first candidates for central dijet production with both leading protons tagged at the LHC, using the combined CMS and TOTEM detectors.
\end{abstract}

XXI International Workshop on Deep-Inelastic Scattering and Related Subjects 22-26 April, 2013

Marseilles, France

\footnotetext{
* Speaker.
} 


\section{Introduction}

Diffractive interactions can be characterized by the presence of at least one non-exponentially suppressed large rapidity gap (LRG) in the final state, defined as a region in pseudorapidity devoid of particles. The origin of LRGs can be attributed to a color-singlet exchange carrying vacuum quantum numbers, commonly referred to as Pomeron $(\mathbb{P})$ exchange.

Soft ${ }^{1}$ diffractive interactions cannot be calculated within perturbative quantum chromodynamics (pQCD), and traditionally have been described by models based on Regge theory. The predictions of these models generally differ when extrapolated from pre-LHC center-of-mass energies $(\sqrt{s} \leq 1.96 \mathrm{TeV})$ to $7 \mathrm{TeV}$ at LHC. Thus, measurements of diffractive cross sections at the LHC provide a valuable input for understanding diffraction and improving the modeling of diffraction in current event generators. Diffractive processes involving a hard parton-parton scattering are characterized by the presence of high- $p_{T}$ jets in the final state in addition to the rapidity gap. Such processes can be calculated within $\mathrm{pQCD}$, however experimental data are sensitive to soft rescattering between spectator partons, which significantly suppresses the formation of the rapidity gap. Measurements of hard diffraction at the LHC, in addition to testing the pQCD description of diffractive exchanges, provide also important information on gap survival probability.

This paper reviews recent results on diffractive processes obtained by the CMS experiment at the LHC. The first CMS measurements of single-diffractive (SD), double-diffractive (DD), and forward rapidity gap cross sections, as well as diffractive production of dijets at $\sqrt{s}=7 \mathrm{TeV}$ are presented. Results are based on 2010 data when the LHC was running in a low pile-up scenario, most suitable for event selection based on the LRG signature. In addition, event displays of dijet candidates with two leading protons at $\sqrt{s}=8 \mathrm{TeV}$, obtained from a combined CMS/TOTEM analysis performed on a common data collected in low-pileup runs in 2012, are presented.

\section{Inclusive diffractive cross sections}

Diffractive cross sections have been measured [1] based on a minimum-bias sample at $\sqrt{s}=7 \mathrm{TeV}$. The SD and DD events (Fig. 1) were separated using the CASTOR calorimeter, which covers the very forward region of the experiment, $-6.6<\eta<-5.2$. Minimumbias events were selected by requiring a signal in any of the BSC (Beam Scintillator
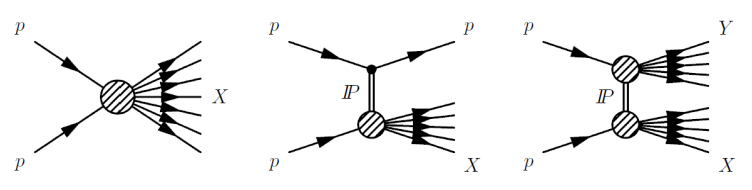

Figure 1: Schematic diagrams of (a) non-diffractive, $p p \rightarrow X$, and diffractive processes with (b) singledissiociation, $p p \rightarrow p X$, and (c) double-dissociation, $p p \rightarrow X Y$. The $X(Y)$ represents a dissociated-proton. Counter) devices and the presence of at least two energy deposits in the central detector $(|\eta| \lesssim 4.7)$. No vertex requirement was imposed. Diffractive events were selected by requiring the presence of a forward rapidity gap reconstructed at the edge of the central detector or central gap. The forward gap on the positive (negative) side was reconstructed in terms of the variable $\eta_{\max }\left(\eta_{\min }\right)$ defined as the highest (lowest) $\eta$ of a particle reconstructed in the detector. The central gap was reconstructed as $\Delta \eta^{0}=\eta_{\max }^{0}-\eta_{\min }^{0}$, with $\eta_{\max }^{0}\left(\eta_{\min }^{0}\right)$ defined as the closest-to-zero $\eta$ of a particle reconstructed on the positive (negative) $\eta$-side of the central detector, with an additional requirement of activity on both sides of

\footnotetext{
${ }^{1}$ with no hard scale, which is needed for perturbative calculations.
} 

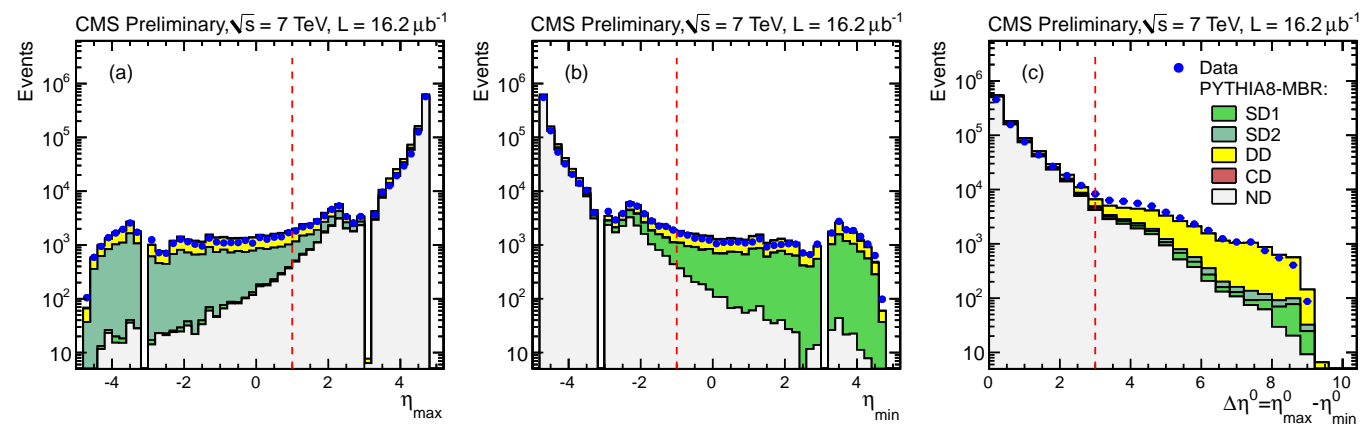

Figure 2: Distributions of (a) $\eta_{\max }$, (b) $\eta_{\min }$ and (c) $\Delta \eta^{0}=\eta_{\max }^{0}-\eta_{\min }^{0}$, compared to predictions of a PYTHIA 8-MBR simulation normalized to the luminosity of the data. Contributions from each of the generated processes are shown separately. The DD MC events are additionally scaled downwards by $15 \%$.

the detector. Figure 2 shows the distributions of $\eta_{\max }, \eta_{\min }$ and $\Delta \eta^{0}$ compared to predictions of PYTHIA8-MBR [2,3]. Diffractive events appear as a flattening of the exponentially falling distributions (non-diffractive events, ND, with rapidity gaps due to random multiplicity fluctuations), and dominate the regions of low $\eta_{\max }$, high $\eta_{\min }$, or high $\Delta \eta^{0}$.

The event sample after the $\eta_{\min }>-1$ selection (Fig. 2, middle) was used to extract SD and DD cross sections. The sample consists of approximatelly equal numbers of SD and DD events for which one of the dissociated masses is low and escapes detection in the central detector. Subsamples enhanced in SD and DD events were selected by requiring an absence or a presence of an energy deposit (above threshold) in the CASTOR calorimeter. The SD/DD separation with CASTOR is presented in Fig. 3, showing the distribution of the variable $\xi$ calculated from all energy deposits in the detector. For SD events, $\xi$ approximates the incomingproton momentum loss. These distributions were used to measure the differential SD cross section as a function of $\xi$, and the differential DD cross section as a function of $\xi_{X}=M_{X}^{2} / \mathrm{s}$ for $0.5<\log _{10}\left(M_{Y} / \mathrm{GeV}\right)<1.1$ (CASTOR acceptance), after subtracting the background contribution to the signal (DD to SD and ND to DD). Results are compared to MC models in Figs. 5 (left) and (middle), respectively. The predictions of PYTHIA8-MBR are shown for two values of the $\varepsilon$ parameter of the Pomeron trajectory $\left(\alpha(t)=1+\varepsilon+\alpha^{\prime} t\right), \varepsilon=0.08$ and $\varepsilon=0.104$. Both values describe the measured SD cross section within uncertainties, while the DD data favor the smaller value of $\varepsilon$. The predictions of PYTHIA8-4C and PYTHIA6 describe well the measured DD
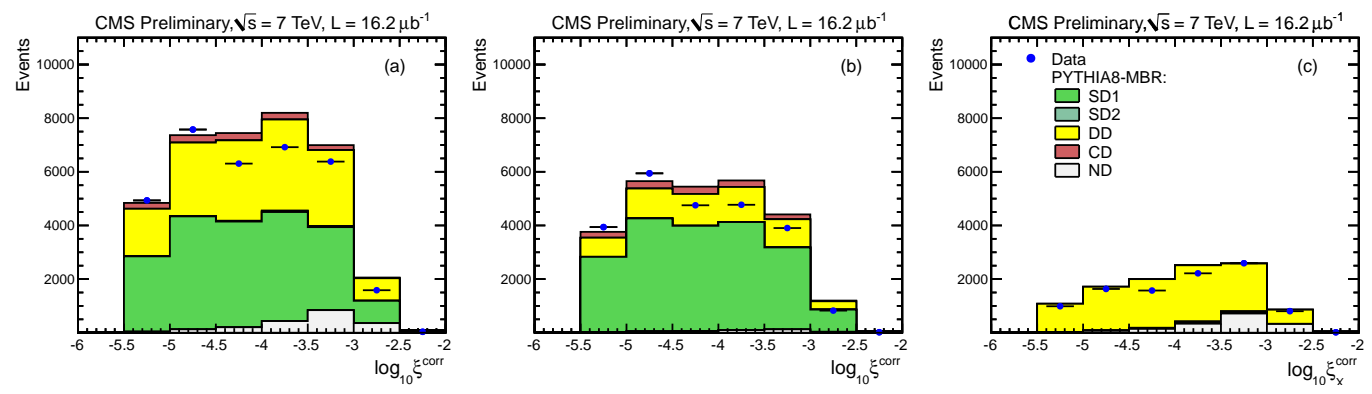

Figure 3: Detector-level distributions of $\xi$ for (a) the events after the $\eta_{\min }>-1$ selection, and its subsamples corresponding to (b) the absence and (c) the presence of an energy deposit in CASTOR. The data are compared to predictions of the PYTHIA8-MBR simulation. 

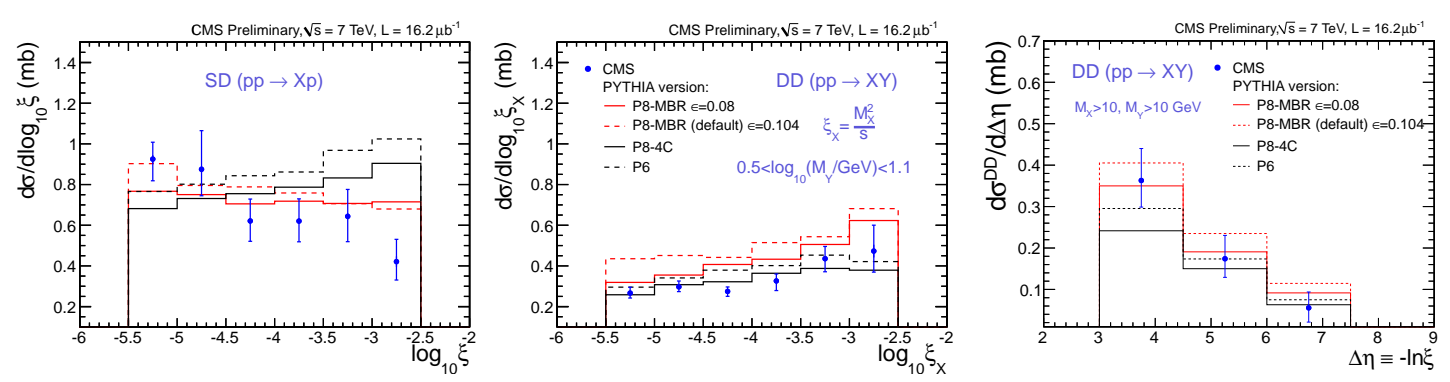

Figure 4: The SD (left) and DD (middle) cross sections as a function of $\xi$, and the DD cross section as a function of $\Delta \eta$ (right), compared to PYTHIA6, PYTHIA8-4C and PYTHIA8-MBR MC. Error bars are dominated by systematic uncertainties (HF calorimeter energy scale, and hadronization and diffraction model).

cross section, but fail to describe the falling behavior of the data. The total SD cross cross section integrated over the region $-5.5<\log _{10} \xi<-2.5\left(12 \lesssim M_{X} \lesssim 394 \mathrm{GeV}\right)$ was measured to be $\sigma_{v i s}^{S D}=4.27 \pm 0.04$ (stat.) ${ }_{-0.58}^{+0.65}$ (syst.) $\mathrm{mb}$ (dissociation of either proton).

The event sample after the $\Delta \eta^{0}>3$ selection (Fig. 2, right) was used to extract the differential DD cross section as a function of the central-gap width, $\Delta \eta$. The cross section for $\Delta \eta>3, M_{X}>$ $10 \mathrm{GeV}$ and $M_{Y}>10 \mathrm{GeV}$ is presented in Fig. 4 (right), showing a reasonable agreement with MC predictions. The total DD cross cross section integrated over this region was measured to be $\sigma_{\text {vis }}^{D D}=0.93 \pm 0.01$ (stat. $)_{-0.22}^{+0.26}$ (syst.) mb.

The inclusive differential cross section for events with a forward rapidity gap, $\mathrm{d} \sigma / \mathrm{d} \Delta \eta^{F}$, was measured as well [1], with a method originally proposed in Ref. [4] and used in the ATLAS measurement [5]. The forward rapidity gap was defined as the larger of gaps on both sides of the CMS detector, $\Delta \eta^{F}=\max \left(4.7-\eta_{\max }, 4.7+\eta_{\min }\right)$. A Bayesian-unfolded and fully corrected cross section for particles with $p_{T}>200 \mathrm{MeV}$ and $|\eta|<4.7$ was extracted and compared to Pythia6Z2*, Pythia8-4C and Pythia8-MBR ( $\varepsilon=0.08$ and 0.104$)$ predictions. Figure5 (left) shows the comparison to the Pythia8-MBR $(\varepsilon=0.08)$ simulation which desribes the data best. A comparison to the ATLAS measurement [5] is shown in Fig. 5 (right). The CMS measurement extends
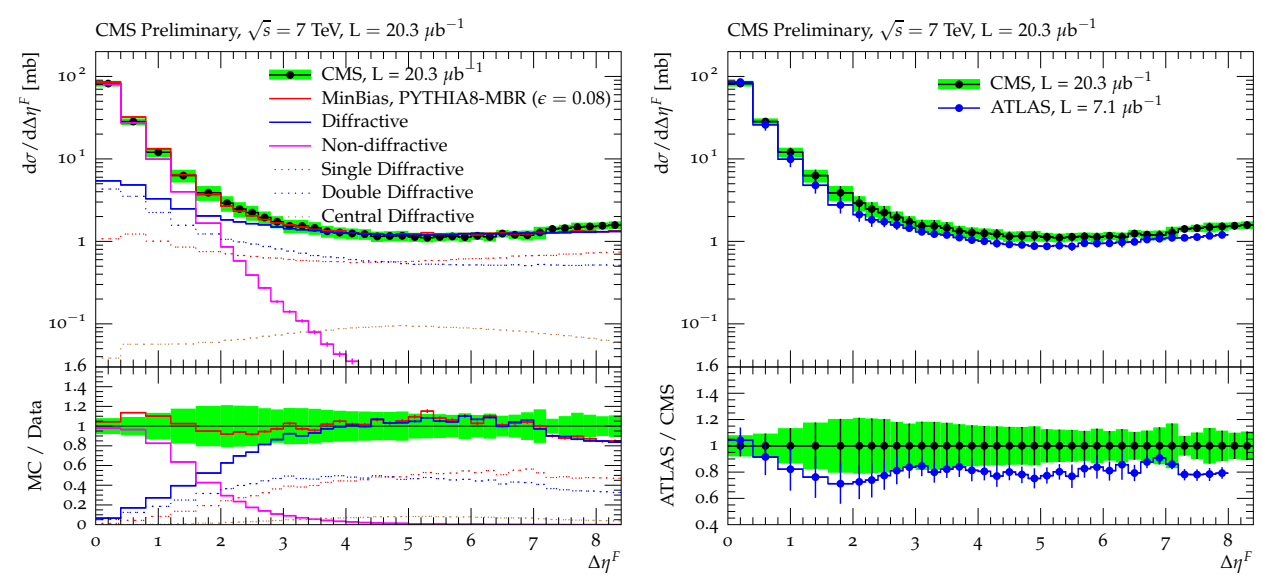

Figure 5: Differential cross section for events with a forward rapidity gap , $\mathrm{d} \sigma / \mathrm{d} \Delta \eta^{F}$, for stable particles with $p_{T}>200 \mathrm{MeV}$ and $|\eta|<4$.7, compared with: (left) the hadron level prediction of PyTHIA8-MBR $(\varepsilon=0.08)$, and (right) the ATLAS measurement [5] ( $\left.p_{T}>200 \mathrm{MeV},|\eta|<4.9\right)$. 
the ATLAS result by 0.4 unit of gap width. Although a different hadron level definition and different MC samples were used for unfolding, both results agree within uncertainties (dominated by HF energy scale, and hadronization and diffraction model uncertainties in the CMS measurement).

\section{Diffractive dijet production}

Diffraction with a hard scale has been studied using events dominated by a contribution from diffractive dijet production [6] (Fig. 6). An inclusive dijet sample at $\sqrt{s}=7 \mathrm{TeV}$ has been selected by requiring at least two jets with $p_{T}>20 \mathrm{GeV}$ and $|\eta|<4.4$, reconstructed using the anti- $k_{T}$ algorithm with a radius $R=0.5$. Dijet events have been studied as a function of the variable $\widetilde{\xi}$, calculated from all energy deposits in the detector (above threshold). Diffractive events populate the region of low values of $\widetilde{\xi}$, which for the SD

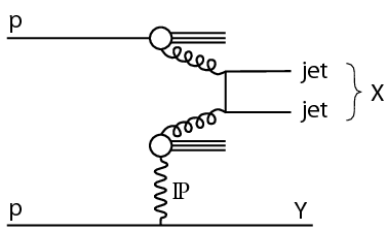

Figure 6: Schematic diagram of diffractive dijet production. process approximates the fractional momentum loss of the incoming proton.

The relation between $\tilde{\xi}$ and a LRG, defined by requiring no individual energy deposits above $4 \mathrm{GeV}$ in one of the two HF calorimeters $(3<|\eta|<5)$, is illustrated in Fig. 7 (left), showing the distribution of events as a function of $\tilde{\xi}$ before (full dots) and after (open dots) applying the LRG condition. The data are well described by a combination of SD (POMPYT) and ND (PYTHIA6$\mathrm{Z2}$ ) MC samples, whose relative yields were obtained from a fit to the $\widetilde{\xi}$ distribution in the inclusive dijet sample. Cross sections for inclusive dijet production, corrected to the stable-particle level, are shown in Fig. 7(right) for $\widetilde{\xi}<0.01$ in three bins of $\widetilde{\xi}$. Results are compared to ND (PYTHIA6 and PYTHIA8) and diffractive (POMPYT SD, POMWIG SD and PYTHIA8 SD+DD) MC models, as well as to NLO calculations of the SD cross section from POWHEG. An excess of events in the low- $\widetilde{\xi}$ region w.r.t. non-diffractive samples is clearly seen. In the lowest $\widetilde{\xi}$ bin $(0.0003<\widetilde{\xi}<0.002)$ the diffractive PYTHIA8 prediction is a factor of $\sim 2$ below the data. The LO predictions from POMPYT and POMWIG, and NLO predictions from POWHEG (all three based on dPDFs extracted from HERA experiments) overestimate the data by a factor of $\sim 5$ and $\sim 10$, respectively. After correcting for the DD contribution, these factors can be converted into an estimate of the gap survival probability, which ranges from $\approx 0.08 \pm 0.04$ (NLO) to $\approx 0.12 \pm 0.05$ (LO).
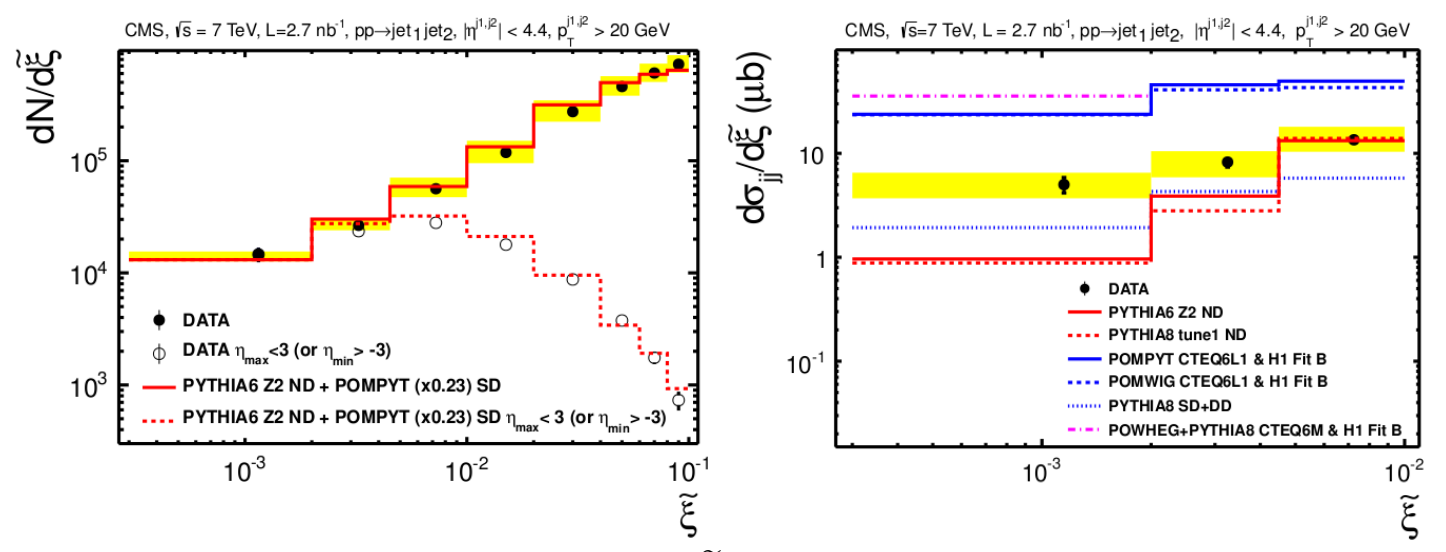

Figure 7: The distribution of events as a function of $\widetilde{\xi}$ obtained before and after the LRG requirement (left), and the differential cross section as a function of $\widetilde{\xi}$ (right) for dijet production compared to MC predictions. 


\section{CMS+TOTEM event displays of high- $p_{T}$ jets with two leading protons}

During the 2012 low-pileup 90m $\beta^{*}$ LHC operation CMS and TOTEM participated in a common data taking. Each experiment took data separately with a bi-directional exchange of CMS and TOTEM trigger information. Offline, events were synchronised in terms of orbit and bunch number matching. A preliminary analysis of high- $p T$ jet production with the proton tags, $p p \rightarrow p p+j e t s$ (Fig. 9 for dijets), has been performed. Event selection in CMS

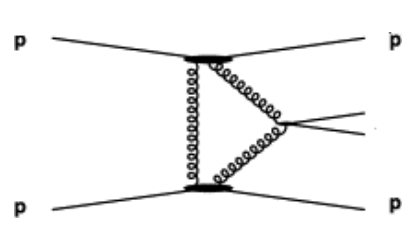

Figure 8: Schematic diagram of exclusive dijet production. required at least two jets with $p_{T}>20 \mathrm{GeV}$ and $|\eta|<4.4$, reconstructed using the anti- $k_{T}$ algorithm with a radius $R=0.5$. In addition, reconstructed proton tracks in the TOTEM Roman Pot detectors on both sides of the interaction point were required, in coincidence with no activity present in the CMS Forward Shower Counters (FSC, $6<|\eta|<8$, installed in 2012). Event displays of dijet and multi-jet events which passed the selection criteria can be found in Ref. [7]. An example of such an event, with three jets of $p_{T}=65,45$ and $27 \mathrm{GeV}$, and a hadronic mass of $244 \mathrm{GeV}$ (TOTEM) or $219 \mathrm{GeV}$ (CMS), is shown in Fig. 9.

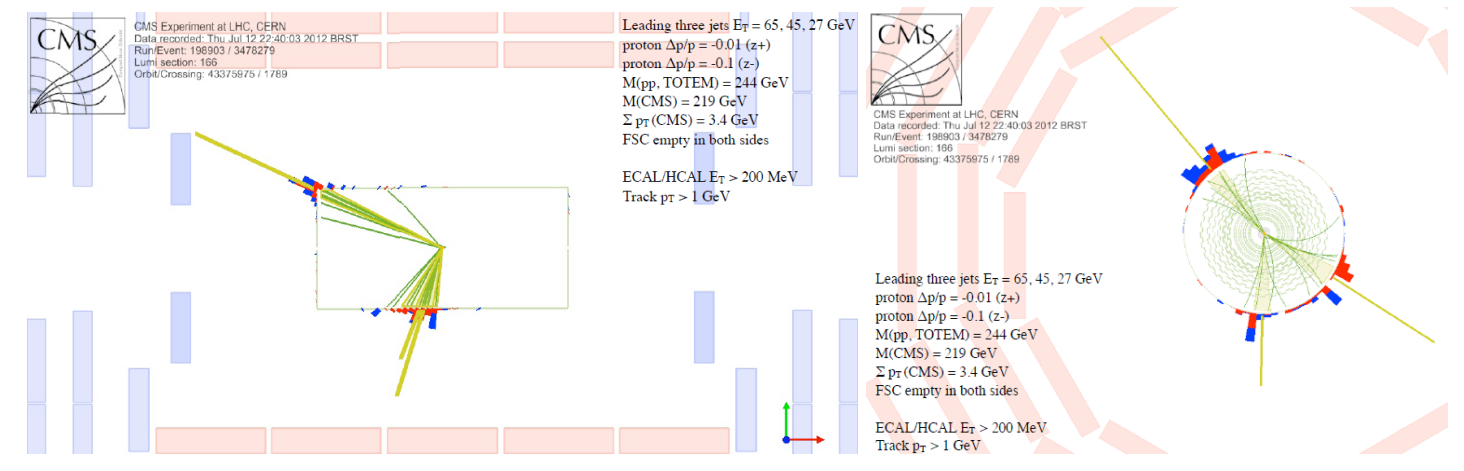

Figure 9: An event desplay of high- $p_{T}$ jets with leading protons detected in the TOTEM RPs.

\section{References}

[1] CMS Collaboration, Measurement of diffraction dissociation cross sections at $\sqrt{s}=7$ TeV at the LHC, CMS-FSQ-12-005.

[2] R. Ciesielski, K. Goulianos, MBR Monte Carlo Simulation in PYTHIA8, arXiv:1205.1446.

[3] K. Goulianos, Hadronic diffraction: where do we stand?, arXiv:hep-ph/0407035; K. Goulianos, Diffraction in QCD, arXiv:hep-ph/0203141; K. Goulianos, A New Statistical Description of Hadronic and $e^{+} e^{-}$Multiplicities., Phys. Lett.B193 (1987) 1.

[4] E. Nurse, S. Sen, Methods to Select Soft Diffraction Dissociation at the LHC, arXiv:1107.2688.

[5] ATLAS Collaboration, Rapidity gap cross sections measured with the ATLAS detector in pp collisions at $\sqrt{s}=7 \mathrm{TeV}$, Eur. Phys. J. C72 (2012) 1926 [arXiv:1201.2808].

[6] CMS Collaboration, Observation of a diffractive contribution to dijet production in proton-proton collisions at $\sqrt{s}=7$ TeV, Phys. Rev. D 87 (2013) 012006 [arXiv:1209.1805].

[7] CMS Collaboration, CMS-TOTEM event display: high- $p_{T}$ jets with two leading protons, CMS-DP-2013-004; CMS Collaboration, Central high- $p_{T}$ jet production during low pile-up, high $\beta^{*}$ run at $\sqrt{s}=8 \mathrm{TeV}$, CMS-DP-2013-006. 